\title{
Photoresist Absorption Measurement at Extreme Ultraviolet (EUV) Wavelength by Thin Film Transmission Method
}

\author{
Atif Shehzad $^{1,2, a}$, Yannick Vesters ${ }^{1,3, a}$, Danilo De Simone ${ }^{1 *}$, Ivan Pollentier ${ }^{1}$, \\ Stefano Nannarone ${ }^{4}$, Geert Vandenberghe ${ }^{1}$, and Stefan De Gendt ${ }^{1,3}$ \\ ${ }^{1}$ Imec, Kapeldreef 75, B-3001 Leuven, Belgium \\ ${ }^{2}$ University of Neuchâtel, Institute of Physics, Av. de Bellevaux 51, \\ CH - 2000 Neuchâtel, Switzerland \\ ${ }^{3}$ KU Leuven, Dept. of Chemistry, Celestijnenlaan 200F, B-3001 Leuven, Belgium \\ ${ }^{4}$ Istituto Officina dei Materiali (IOM), Italian National Research Council (CNR), \\ Trieste, Italy \\ ${ }^{a}$ Shehzad and Vesters contributed equally and should both be considered first authors \\ *danilo.desimone@imec.be
}

\begin{abstract}
A methodology to measure photoresist absorption under soft X-ray radiation is presented in this work. The high photoresist sensitivity required for the extreme-ultraviolet lithography (EUVL) technology can be achieved by increasing the EUV photon absorption of photoresist material, hence high extinction coefficient is preferred. To determine the extinction coefficient of a photoresist, a small-scale manufacturing process has been developed to obtain free standing thin silicon nitride ( $\mathrm{SiN}$ ) membranes and use it as sample holder for photoresist thin film characterization. The SiN membrane has been characterized in terms of film thickness, composition and density. Transmission measurements through the thin films of $\mathrm{SiN}$ and photoresist have been carried out in the energy range between $90.6 \mathrm{eV}$ and $92.6 \mathrm{eV}$ by using X-ray Absorption Spectroscopy (XAS) at the BEAR beamline Elettra synchrotron research facility, in Italy. The method permits an experimental measurement of the absorption coefficient of thin films, with an accuracy of $3 \times 10^{-4}$, providing a tool helpful for EUV photoresist research and development.
\end{abstract}

Keywords: EUV Lithography, Photoresist, Absorption, Metrology

\section{Introduction}

The EUV lithography is recognized as the next technology that enables the scaling down of semiconductor devices for high-volume manufacturing [1]. Today the $5 \mathrm{~nm}$ logic technology node (N5) is the first scaling node at which industry will likely insert EUV into production, which will bring a reduction in processing steps and therefore in total cost of ownership [2,3]. To achieve the productivity targets, EUV photoresist materials and their related processes play an important role. The performance of a photoresist for microelectronic fabrication depends on several factors as following. High resolution, high sensitivity, low line-width roughness and defect free pattern are the key requirements for a photoresist in EUVL [4]. In EUV technology, due to the limited reflectivity $(\sim 70 \%)$ of the $\mathrm{Mo} / \mathrm{Si}$ multilayers for EUV optics, the light power delivered at wafer level is strongly reduced compared to the power of the source [5]. Therefore, high sensitivity of the photoresist is strongly needed to reduce the development cost of high-power exposure sources while ensuring sufficient throughput. High EUV photon absorption in photoresist is key to have high photoresist sensitivity. Photon absorption of a material can be theoretically calculated [6], using absorption cross section [7], the exact stochiometric composition of the material and its density. Several methods have been investigated to achieve high photoresist 
sensitivity. For instance, addition of high EUV absorption metal species such as Hafnium (Hf), Zirconium ( $\mathrm{Zr}$ ) [8] or other sensitizers [9] in the base resin of the EUV photoresist. By increasing the EUV absorption of the base resin, secondary electron generation can be increased and thus photoresist sensitivity $[10,10]$. Nevertheless, calculated and measured absorption values are not always perfectly matching, and deviations were reported [9]. Furthermore, the exact composition and density of a photoresist is not always available, especially in the case of resist screening of state-ofthe-art photoresists from different suppliers.

Therefore, it is important to be able to measure accurately the absorption of various photoresists. The standard way of obtaining absorption at a certain wavelength is to measure the transmittance through the sample. Transmittance of a homogenous medium is given by the expression

$$
T(\lambda)=\frac{I(\lambda)}{I_{0}(\lambda)}
$$

where $T$ is the transmittance, $I_{0}$ is the intensity of incident light, $I$ is the transmitted light intensity and $\lambda$ is the wavelength of light being used. As the refractive index of most material, including $\mathrm{SiN}$ and organic materials, is very close to 1 at EUV wavelength, the reflections at interfaces can be neglected compared to the absorption in the medium. Using transmittance, it is thus possible to obtain the absorption coefficient of a material using LambertBeer law

$$
I=I_{0} e^{-\alpha(\lambda) d}
$$

where $d$ is the thickness of the material and $\alpha$ is the wavelength dependent absorption coefficient which is further given as

$$
\alpha=\frac{4 \pi k}{\lambda}
$$

where $k$ is the extinction coefficient. Encountering Eqs. (2) and (3) into Eq. (1) the transmittance is given by

$$
T(\lambda)=e^{-\frac{4 \pi k d}{\lambda}}
$$

Thus, measuring the transmittance of the EUV photoresist material allows the determination of the absorption coefficient, using Eq. (4).

Nevertheless, direct measurement of the transmittance of photoresist materials presents a major challenge. EUV light at $13.5 \mathrm{~nm}$ wavelength is absorbed by all materials. This has a strong impact on the technology: reflective optics instead of transmissive, exposure under vacuum, etc. [12].
It also makes it impossible to measure transmittance of a bulk material. For example, the attenuation length of PMMA is $190 \mathrm{~nm}$ (at EUV wavelength) meaning that only $0.5 \%$ of the light is transmitted through $1 \mu \mathrm{m}$ of material [13]. The transmission of a material should thus be measured through a freestanding thin film $(<100 \mathrm{~nm})$, and relatively large areas $\left(>1 \mathrm{~mm}^{2}\right)$ are required to align the light source, the sample and the detector. But preparing a freestanding film of photoresist polymer is extremely challenging due to the limited mechanical strength of such polymers. Two transmission measurement approaches have been explored, direct photoresist coating onto a photodiode [14] and coating on a thick membrane, with reported thicknesses for the support membrane of $270 \mathrm{~nm}$ [15], $150 \mathrm{~nm}$ [16] and $100 \mathrm{~nm}[17,18]$. In the method presented in this paper, the photoresist is first coated on a thick substrate which is subsequently etched. This allows for very thin membrane after etching, allowing better transmission measurements. The membrane is a stack of a thin silicon nitride film, offering mechanical strength, on top of which the photoresist sample is coated. Large area free-standing films can be achieved with a $30 \mathrm{~nm}$ thick silicon nitride film.

Using such bilayer samples, the transmittance of the stack can be measured, and both absorption coefficients can be calculated back using Eq. 5,

$$
\begin{aligned}
T_{\text {Measured }}(\lambda) & =T_{S i N}(\lambda) \times T_{P R}(\lambda) \\
& =e^{\frac{-4 \pi}{\lambda}\left(k_{S i N} d_{S i N}+k_{P R} d_{P R}\right)}
\end{aligned}
$$

where the index $\mathrm{SiN}$ relates to the silicon nitride membrane layer and the index PR to the photoresist thin film layer.

In this work, we describe the developed set-up for the preparation of such thin samples where a photoresist is placed on top of a SiN membrane. This allows to measure its transmittance in the energy range between $90.6 \mathrm{eV}$ and $92.6 \mathrm{eV}$ (thus around EUV lithography wavelength) by using Xray Absorption Spectroscopy (XAS) at the BEAR beamline Elettra synchrotron research facility, in Italy.

\section{Experimental}

The preparation of the samples was realized in a $300 \mathrm{~mm}$ cleanroom (Imec, Belgium). Samples were prepared from $300 \mathrm{~mm}$ silicon wafers, each wafer containing an array of samples having a frame dimension of $15 \mathrm{~mm}$ by $16.5 \mathrm{~mm}$. The process flow to obtain the final sample is presented in Fig. 1. We started with the deposition of SiN by LPCVD in an 

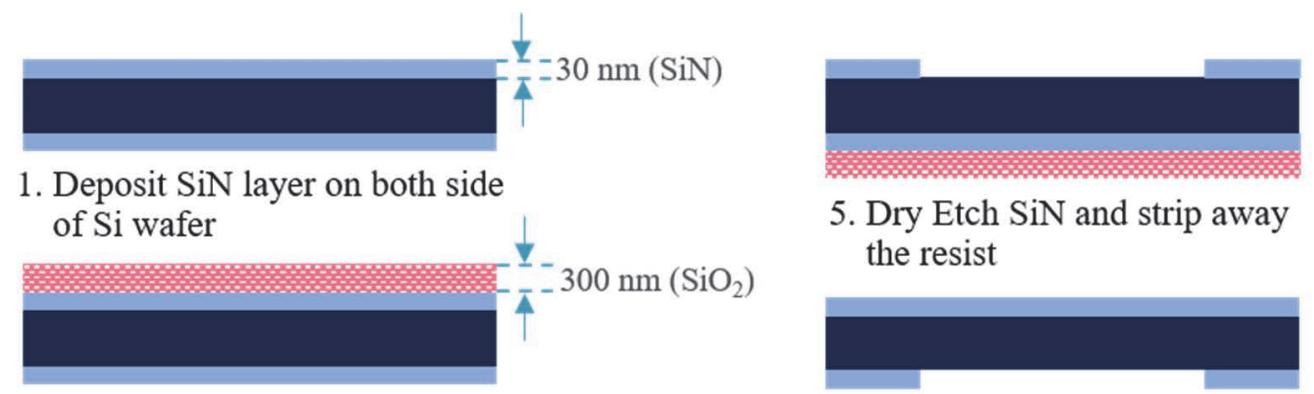

5. Dry Etch SiN and strip away the resist

2. Deposit $\mathrm{SiO}_{2}$ protective layer on front side

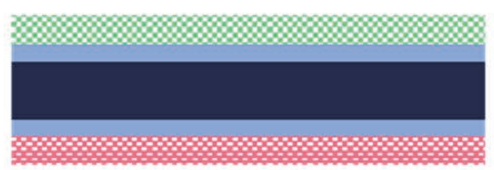

3. Flip the wafer and coat photoresist on back side

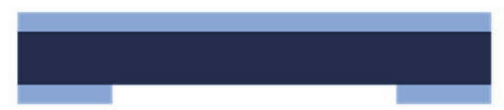

6. Flip the wafer, wet etch $\mathrm{SiO}_{2}$ with $\mathrm{HF}$, dice the wafer
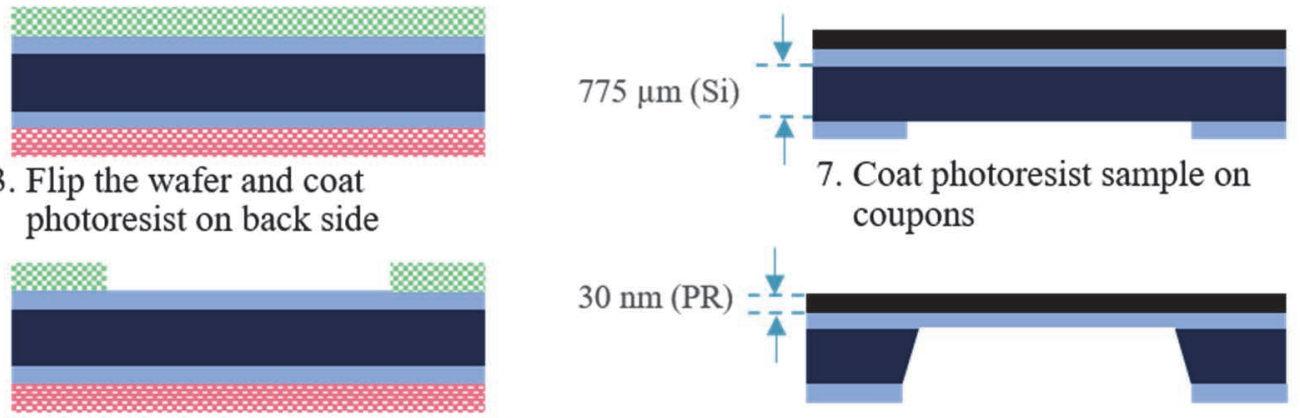

8. Wet etch Si wafer with $\mathrm{KOH}$ to obtain membrane

Fig. 1. Process flow for sample preparation. For step 8, more details in Fig. 3.

ASM A412 furnace (step 1). This step covers both sides of the wafer with a $30 \mathrm{~nm}$ thin Si-rich silicon nitride film. In order to protect and avoid damage of the SiN film by wafer handling robots during further processing steps, a protective film should be placed on the front side. Therefore, a $300 \mathrm{~nm}$ thick $\mathrm{SiO}_{2}$ layer was deposited by CVD using Applied Materials Producer ${ }^{\circledR}$ tool (step 2). The wafer was then flipped and the backside of the wafer was coated with photoresist (step 3) for the lithography step. Using ASML Twinscan XT1000 connected with TEL LITHIUS PRO track, different window sizes in the photoresist $\left(2 \times 2,4 \times 4\right.$ and $\left.8 \times 8 \mathrm{~mm}^{2}\right)$ were printed in this fourth step. The pattern from the lithography represents the final dimension of the $\mathrm{SiN}$ membrane. In process step 5, dry etching of SiN and stripping away of the remaining photoresist was done using a LAM Kiyo tool. Step 6 was the last process step on the full wafer where the $\mathrm{SiO}_{2}$ protective layer was removed by wet etching with hydrofluoric acid (HF) on a Screen FC-3100 tool.

After the wafer preparation with multiple $\mathrm{SiN}$ windows, the $300 \mathrm{~mm}$ wafer (Fig. 2) was diced into coupons of required dimensions $\left(15 \times 16.5 \mathrm{~mm}^{2}\right)$, each coupon containing one window on the backside.

At this stage, the SiN layer on the front side was characterized with different methods, as presented in Table 1.
Spin-coating photoresist on a thin membrane directly would be impossible due to the high stress generated by the spin-coat process that would break the membrane. Therefore, the EUV photoresist aimed for transmittance measurements was spin coated onto the SiN at this process step, when the silicon substrate is still present. Spin coating was done on coupons using HOLMARC HO-TH-05 spin coater with manual dispense of photoresist. After spin-coating on top of the $\mathrm{SiN}$, the resist was baked at $100{ }^{\circ} \mathrm{C}$ for 60 seconds on a hotplate, hence the photoresist was characterized to measure precisely the film thickness. As photoresist suffers shrinkage under electron microscope, the best technique for photoresist film thickness measurement was ellipsometry. The photoresist film

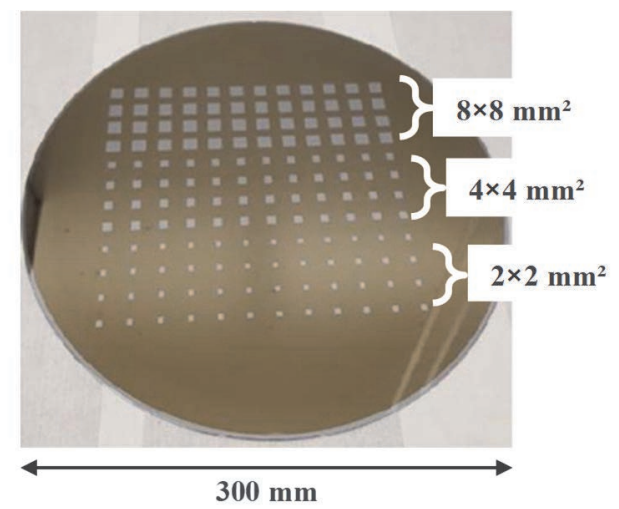

Fig. 2. Picture of the backside of the wafer after step 5. 
Table 1. Tool and method description for the material characterization techniques used on SiN film.

\begin{tabular}{|c|c|c|c|}
\hline & & & \\
\hline Technique & Property & Tool & Details \\
\hline TOF-ERD & Composition & $\begin{array}{l}\text { Customized } \\
\text { Giangrandi } 2007\end{array}$ & $\begin{array}{l}8.0 \mathrm{MeV}^{35} \mathrm{Cl}^{4+} \text { ion beam } \\
39.6^{\circ} \pm 0.5^{\circ} \text { recoil angle }\end{array}$ \\
\hline XRR & $\begin{array}{l}\text { Density } \\
\text { Thickness }\end{array}$ & $\begin{array}{l}\text { PANAlytical X'Pert } \\
\text { Pro diffractometer }+ \\
\text { PIXcel detector }\end{array}$ & $\begin{array}{l}\mathrm{Cu} \mathrm{K} \alpha \text { radiation }(\lambda=0.154 \mathrm{~nm}) \\
\text { Omega step } 0.01^{\circ} \\
\text { Jordan Valley Semiconductors REFS software for data fitting }\end{array}$ \\
\hline XSEM & Thickness & Hitachi SU-8000 & $\begin{array}{l}10 \mathrm{kV} \text { e-beam } \\
400 \mathrm{k} \text { magnification }\end{array}$ \\
\hline $\begin{array}{l}\text { HAADF-STEM } \\
\text { STEM-EDS }\end{array}$ & $\begin{array}{l}\text { Thickness } \\
\text { Composition }\end{array}$ & $\begin{array}{ll}\text { FEI } & \operatorname{Titan}^{3} \text { G2 60- } \\
300 & \end{array}$ & $\begin{array}{l}\text { Sample preparation: covered with spin-on-carbon, and } \mathrm{Pt} \\
\text { capping (Helios } 450 \mathrm{HP} 30 \mathrm{kV} \text { e-beam) before FiB milling } \\
(30 \mathrm{kV} \mathrm{Ga} \text { ion beam) and lift-out }\end{array}$ \\
\hline Ellipsometry & $\begin{array}{l}\text { Thickness } \\
\text { Uniformity }\end{array}$ & KLA Tencor F5 & Harmonic Oscillator model for data fitting \\
\hline
\end{tabular}

was thus characterized using KLA Tencor F5 ellipsometer.

This coating step was finally followed by the membrane formation through etch: potassium hydroxide was selected for its selectivity with a rate of above $500 \mathrm{~nm} / \mathrm{min}$ for $\mathrm{Si}$ versus $0 \mathrm{~nm} / \mathrm{min}$ for silicon nitride [19]. Silicon wet etching with $20 \%$ $(\mathrm{w} / \mathrm{w})$ potassium hydroxide $(\mathrm{KOH})$ solution at $70{ }^{\circ} \mathrm{C}$ was performed for 18 hours in a beaker on a heating plate, monitoring the solution temperature. A dedicated sample holder required to place the backside of the coupon in the etch solution was

(a)

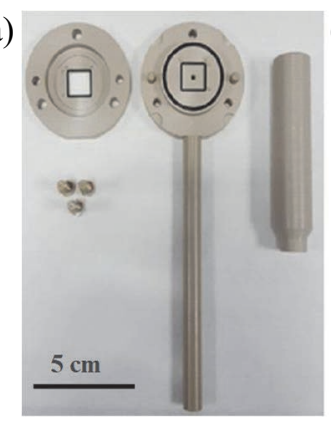

(b)

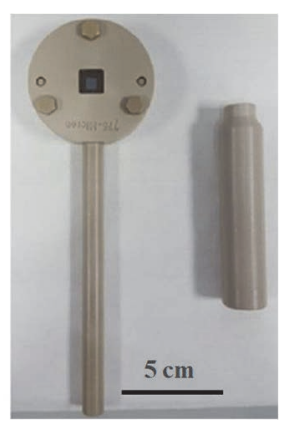

(c)

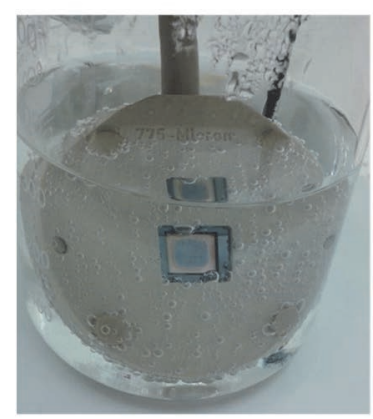

specially designed in such a way that the EUV photoresist on the frontside was fully protected from any contact with the $\mathrm{KOH}$ solution, as visible in Fig. 3.

After etching, final membrane samples were obtained, composed of a bilayer of $\mathrm{SiN}$ and photoresist, as per Fig. 4. These samples were again characterized by ellipsometry in case any changes in the $\mathrm{SiN}$ or photoresist thicknesses would occur during etch.

As described earlier, membranes of different sizes were prepared. While $2 \times 2 \mathrm{~mm}^{2}$ membranes

Fig. 3. Specially designed sample holder (a) before and (b) after mounting the coupon sample. (c) Etching process of the sample in a beaker containing $\mathrm{KOH}$ solution and (d) sketch of the sample while being etched.

(a)

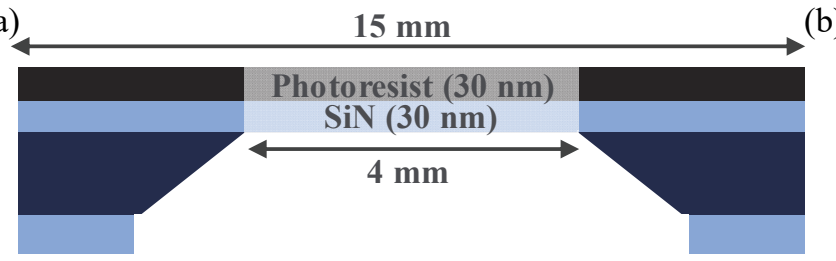

(d)

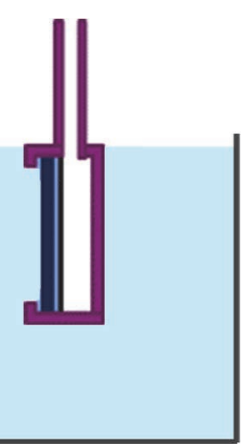

(b)

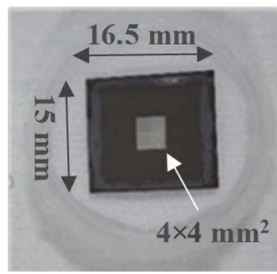

Fig. 4. (a) Layout of the sample after $\mathrm{KOH}$ etch. (b) This membrane sample is ready for EUV transmission measurement. 
are robust and easy to handle, $4 \times 4 \mathrm{~mm}^{2}$ membranes require delicacy and much attention while etching, demounting from the sample holder, handling and shipping. Larger membranes, at $8 \times 8 \mathrm{~mm}^{2}$, are too fragile and break either during the etching step, handling or transport. As the area of $2 \times 2 \mathrm{~mm}^{2}$ is very limited and provide less data points for thickness measurement and transmission measurement, we recommend $4 \times 4 \mathrm{~mm}^{2}$ membranes for such sample preparation.

After sample preparation in Belgium, samples were shipped to Italy for transmission measurement. Special care was taken in shipment preparation to avoid breaking of the fragile membrane samples during transport.

Transmission measurements on membrane samples were carried out at the Bending Magnet for Emission, Absorption and Reflectivity (BEAR) beamline, Elettra Synchrotron Light Laboratory, Italy. The light-transport optics, monochromatization optics and the experimental endstation are under ultra-high vacuum (UHV) environment. Samples were inserted into the chamber onto a sample holder so that light beam (spot dimension about $300 \times 300 \mu \mathrm{m}^{2}$ ) was impinging the membrane surface at normal incidence. The properties of the synchrotron light can be modified according to the requirements. The spectral range selected for the experiment was 90.6$92.6 \mathrm{eV}$. Purity radiation in the range $85-100 \mathrm{eV}$ is accomplished with a Si filter and energy resolution of $0.10 \mathrm{eV}$ is achieved. Transmitted intensity $(I)$ and incoming photon intensity $\left(I_{0}\right)$ were measured with a photodiode SXUV-100 having sensitive area of $10 \times 10 \mathrm{~mm}^{2}$. Energy calibration was done by using the energy cutoff of the Si filter used for higher harmonics rejection. The reflection at the vacuumsample and SiN-photoresist interfaces was considered negligible.

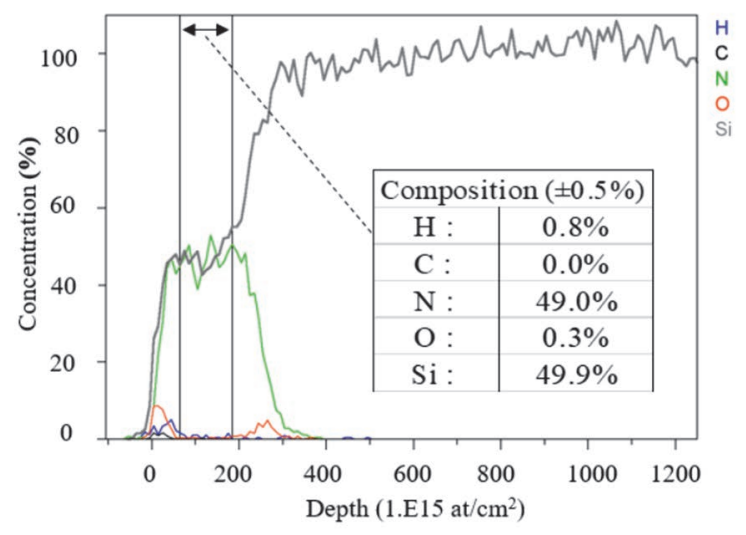

Fig. 5. TOF-ERD results provide the composition of the $\mathrm{SiN}$ membrane as $\mathrm{SiN}_{0.98}$.

\section{Results and discussion}

The accuracy of the absorption measurements will be validated by comparing theoretical and measured absorption for the SiN membrane. The theoretical absorption coefficient of a material can be calculated from its composition and density, therefore we determined the density and composition of the SiN layer through different techniques, after dicing the wafer into coupons (Fig. 1, step 6). We performed composition measurements both by TOF-ERD and by TEM-EDS. The TOF-ERD results are presented in Fig. 5. As calculated from the imbedded table, the center of the silicon nitride film has a composition close to $\mathrm{SiN}_{0.98}$ (other atoms are considered negligible). Additionally, on both sides of the SiN a partially oxidized layer is observed at the interfaces (both the air-SiN interface and the SiN-Si interface).

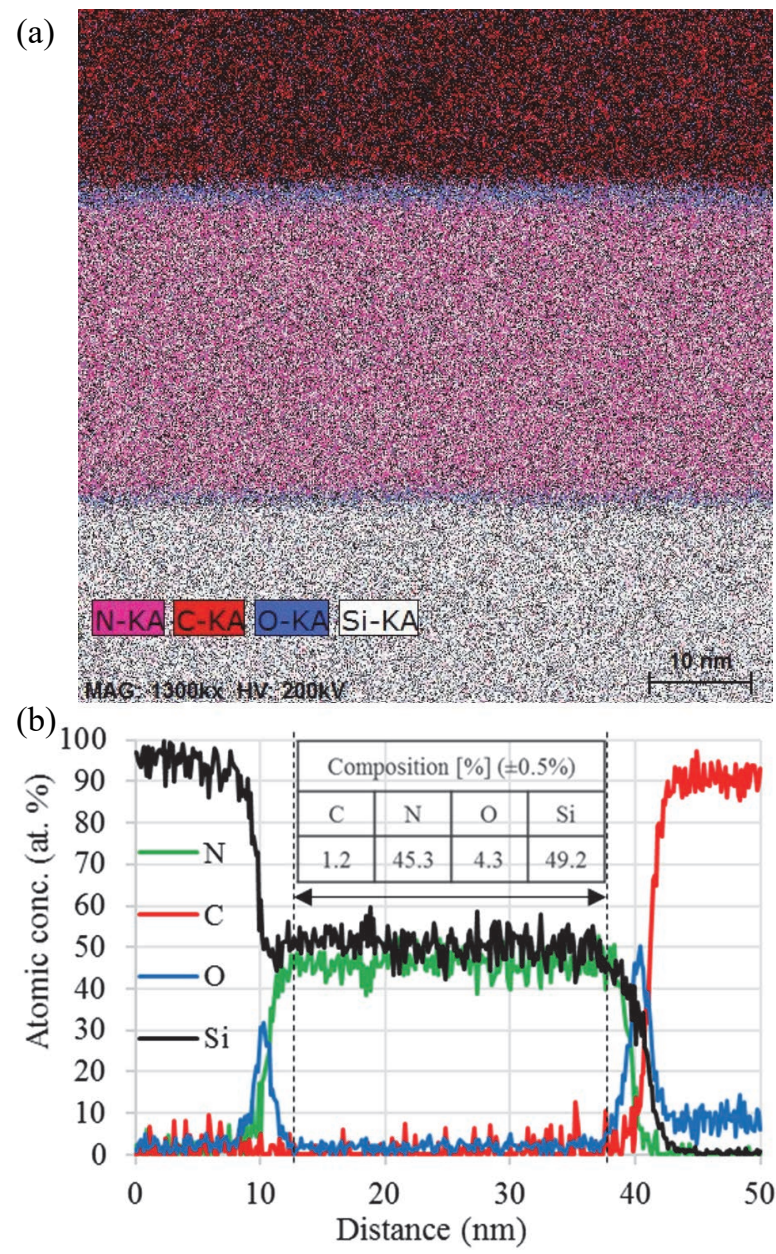

Fig. 6. SiN layer on top of the Silicon substrate: STEMEDS image (a) and corresponding elemental composition graph (b).

The STEM-EDX measurement presented in Fig. 6 provides the composition for the bulk of the film as $\mathrm{SiN}_{0.92} \mathrm{O}_{0.08}$, and presence of oxygen is also 
visible at the interfaces at both side of the silicon nitride layer. As no native oxide etch was realized before SiN deposition, it was expected to observe an oxide interface at the $\mathrm{Si} / \mathrm{SiN}$ interface. The oxide at the air/SiN interface is due to ambient oxidation. As no further treatment is realized before coating the photoresist on the sample, the oxide layer on the top of the sample will still be present during the transmission measurement. There will also be a bottom oxide layer after $\mathrm{KOH}$ etch, generated either at the end of the etch or through ambient oxidation during transport.

Table 2. Comparison of the thicknesses obtained by ellipsometry and electron microscopy.

\begin{tabular}{|l|c|c|}
\cline { 2 - 3 } \multicolumn{1}{c|}{} & $\begin{array}{c}\text { Thickness by } \\
\text { ellipsometry }\end{array}$ & $\begin{array}{c}\text { Thickness by electron } \\
\text { microscopy }\end{array}$ \\
\hline Coupon 1 & $30.2 \pm 0.2 \mathrm{~nm}$ & $30.3 \pm 0.5 \mathrm{~nm}$ (XSEM) \\
\hline Coupon 2 & $29.8 \pm 0.1 \mathrm{~nm}$ & $\begin{array}{c}31.5 \pm 0.2 \mathrm{~nm} \\
\text { (HAADF-STEM) }\end{array}$ \\
\hline
\end{tabular}

(a)

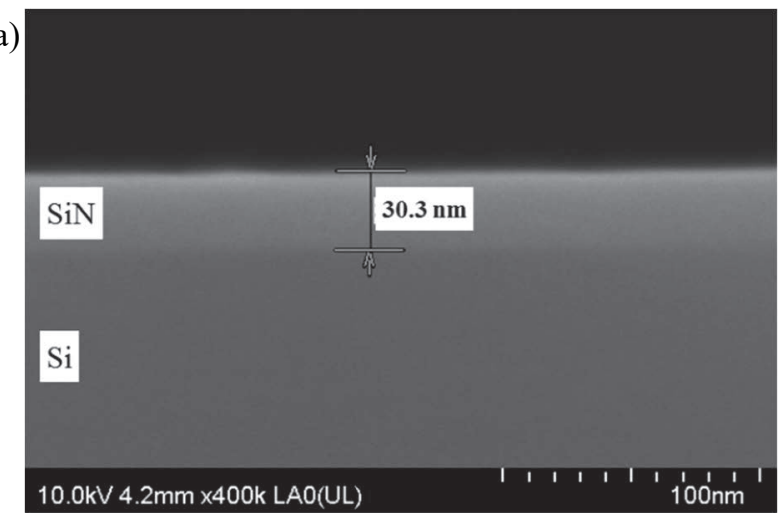

(b)

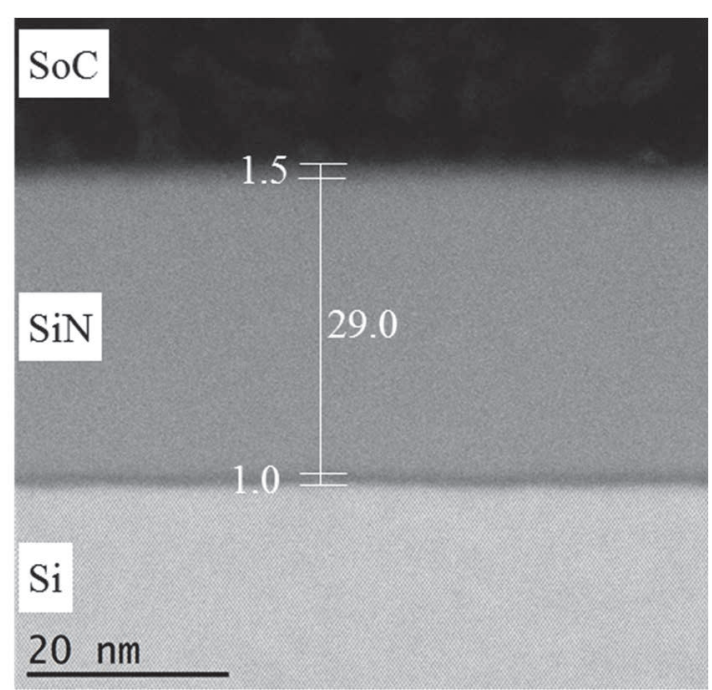

Fig. 7. Thickness of the SiN film measured by X-SEM (a) and HAADF-TEM (b).
As the final calculation of the extinction coefficient of the photoresist is highly dependent on the thickness of both the SiN layer and the photoresist layer, an accurate measurement of this thickness is mandatory. While for future absorption measurement the objective is to measure both thicknesses by ellipsometry only, the accuracy of the SiN thickness measurement was benchmarked by XSEM and HAADF-TEM. Using a harmonic oscillator model considering a single layer of silicon nitride, the thickness obtained by ellipsometry is reported in Table 2, with average value and standard deviation for five points measured. The second columns report the thickness measured from the images in Fig. 7. Whereas the contrast between Si and $\mathrm{SiN}$ is faint in XSEM, the HAADF-STEM offers better differentiation, allowing to measure the thicknesses of the oxide interfaces. The STEM gives a total thickness of $31.5 \mathrm{~nm}$ but $2.5 \mathrm{~nm}$ are associated with the top and bottom oxide interfaces. From this data, the ellipsometry model used for SiN characterization is considered satisfactory with an error evaluated at $1 \mathrm{~nm}$.

Finally, density was evaluated using XRR. The XRR data is presented in Fig. 8. The measured XRR data is fitted to a simulation by adjusting the parameters (thickness, density and roughness) of a structural tri-layer model targeting to differentiate the oxide interfaces and the bulk. XRR fitting calculation is based on a differential evolution algorithm [20], in which an error function is minimized. The total thickness obtained for the membrane was $32.8 \mathrm{~nm}$ with in total $5.4 \mathrm{~nm}$ of oxide at the interface, slightly overestimating the dimensions compared to the TEM images. The density of the bulk was evaluated at $2.94 \pm 0.06 \mathrm{~g} / \mathrm{cm}^{3}$, which is significantly lower than the density of bulk silicon nitride $\mathrm{Si}_{3} \mathrm{~N}_{4}\left(3.17 \mathrm{~g} / \mathrm{cm}^{3}\right)$. From the high-resolution STEM image presented on Fig. 7, where the lattice of the $\mathrm{Si}$ crystalline substrate is visible, it can be seen that SiN layer is an amorphous layer, which explains the lower density compared to crystalline $\mathrm{Si}_{3} \mathrm{~N}_{4}$.

Once the SiN layer was characterized, the sample could be etched (step 8). Two SiN membrane samples, with window size $4 \times 4 \mathrm{~mm}^{2}$, were prepared for transmission measurements: One without photoresist (sample A) and the other coated with photoresist (sample B). After the last etching step, the membrane thickness of the samples was measured using an optimized ellipsometry model (adding a Cauchy model for the photoresist layer) at 16 different points uniformly distributed on 


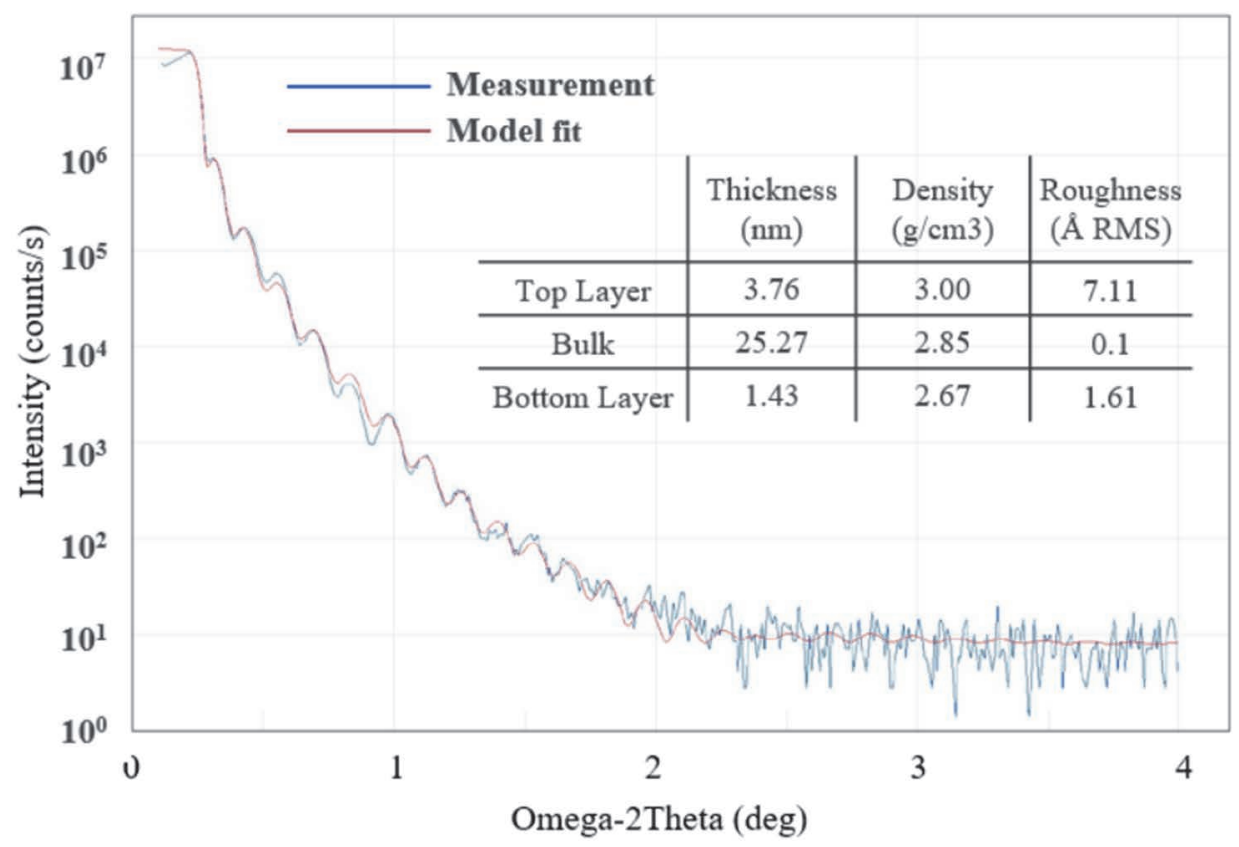

Fig. 8. XRR data and fitted curve for the SiN layer on silicon. Embedded table shows the thickness, density and roughness values obtained with a 3-layer fitting model.

membrane area of $4 \times 4 \mathrm{~mm}^{2}$. In Fig. 9, thickness measurement of SiN membranes and photoresist before and after the etching step are shown. The data was used for estimating the thickness uniformity and the impact of etching. From Fig. 9, it can be seen that the thickness of SiN varies in a relatively low range of $0.7 \mathrm{~nm}$ across the membrane. The SiN film deposited on silicon and as a free-standing membrane give similar thicknesses, which was expected as $\mathrm{SiN}$ etch rate in $\mathrm{KOH}$ is nil. It is thus confirmed that the etch process does not significantly change the SiN membrane thickness.

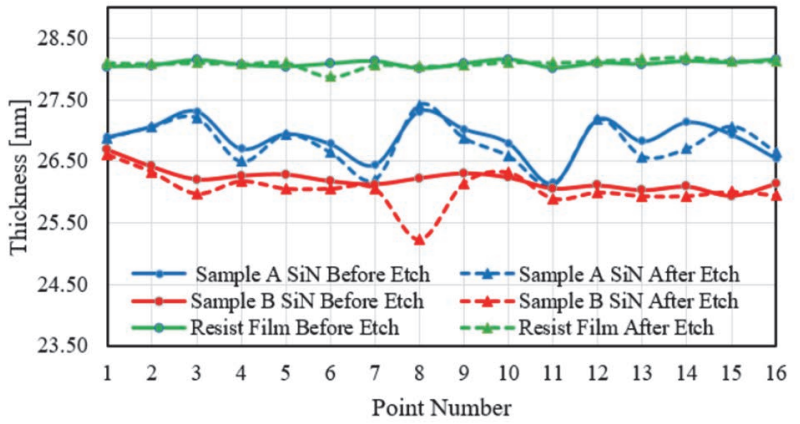

Fig. 9. Result of ellipsometry measurement: Variation in SiN film thickness and photoresist film thickness before (circles) and after (triangles) etching step.

As exposure to ellipsometry light might slightly change the photoresist characteristics, the transmittance measurement was performed in an area of the membrane that was not used for ellipsometry measurement. Nevertheless, we need precise thickness measurement of the spot where transmittance measurement will be performed. Therefore, an interpolation of 16 ellipsometer points was realized (Fig. 10) to obtain thicknesses at 8 unexposed position, where the transmission measurement can take place.
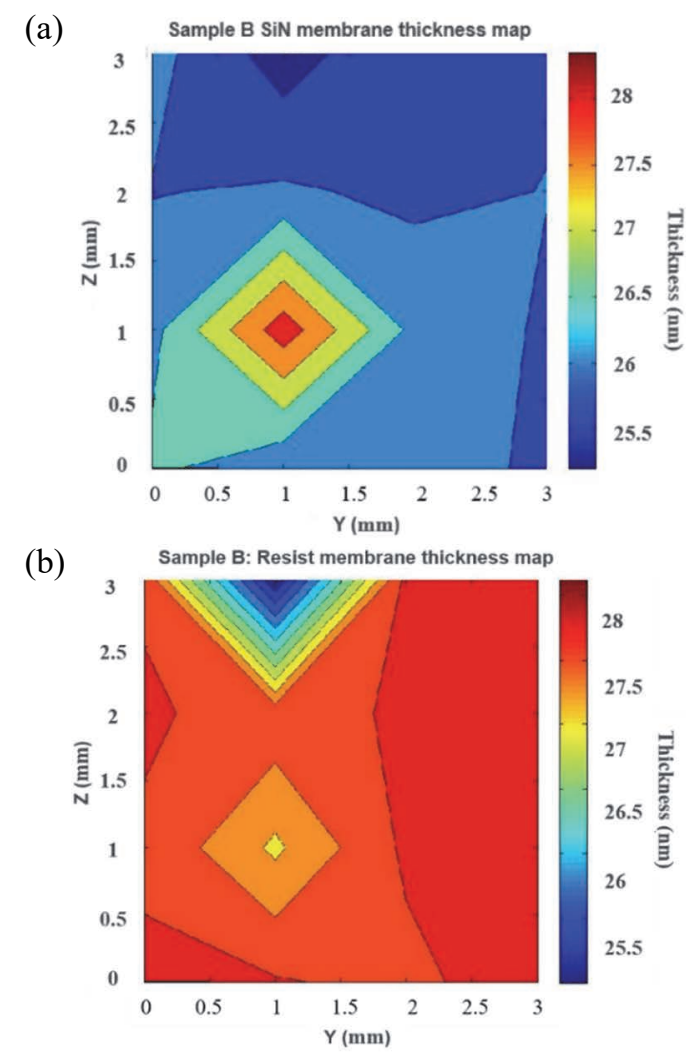

Fig. 10. Result of ellipsometry measurement: interpolated thickness of $\mathrm{SiN}$ membrane (a) and photoresist film (b) for sample B. 
Finally, transmittance from $90.6 \mathrm{eV}$ to $92.6 \mathrm{eV}$ has been measured in steps of $0.1 \mathrm{eV}$ for both samples at the 8 unexposed positions. In both cases, interferences and reflectivity effects at the silicon nitride-vacuum, at the photoresist-vacuum and at the photoresist-silicon nitride have been neglected. Transmission results for sample $\mathrm{A}$ and $\mathrm{B}$ are shown in Fig. 11. At $91.6 \mathrm{eV}$, corresponding to a $13.53 \mathrm{~nm}$ EUV wavelength, the transmission of the $\mathrm{SiN}$ varies between 0.827 and 0.837 depending on the position. The sample with SiN and photoresist has a lower transmission value, in the range 0.751 to 0.757 , which is logical as the photoresist on top of the membrane in sample B is responsible for the extra absorption compared to sample A (membrane only).

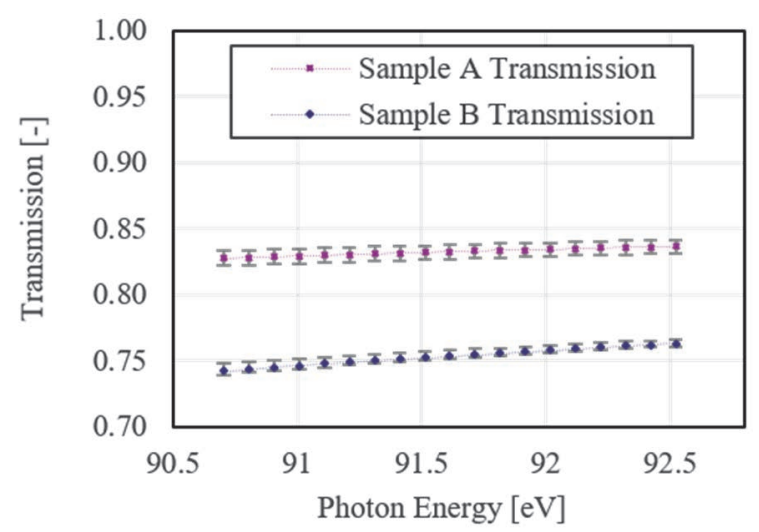

Fig. 11. Transmittance measurement results for sample A (SiN membrane) and sample $\mathrm{B}(\mathrm{SiN}+$ photoresist film).

Extinction coefficient for $\mathrm{SiN}\left(k_{\mathrm{SiN}}\right)$ and photoresist $\left(k_{\mathrm{PR}}\right)$ have been evaluated using the transmittance measurement data. For sample A, the extinction coefficient of $\mathrm{SiN}$ was calculated using Eq. (4) and a thickness $d_{\text {SiN }}$ obtained by ellipsometry (Fig. 9). At $91.6 \mathrm{eV}$, it gives $k_{S i N}=7.4 \pm 0.2 \times 10^{-3}$. The uncertainty was calculated from the propagation of the uncertainties of the ellipsometry and transmission measurements. To assess the accuracy of our experimental measurement procedure, this extinction coefficient was compared with the tabulated data from the Center for X-Ray Optics (CXRO) [7,21].

As visible in Fig. 12, the extinction coefficient of crystalline silicon nitride $\left(\mathrm{Si}_{3} \mathrm{~N}_{4} 3.17 \mathrm{~g} / \mathrm{cm}^{3}\right)$ is not matching correctly the data. But using the density of $2.94 \mathrm{~g} / \mathrm{cm}^{3}$ (as per XRR result), and the exact composition of the film, either $\mathrm{SiN}_{0.98}$ (as per TOFERD result) or $\mathrm{SiN}_{0.92} \mathrm{O}_{0.8}$ (as per STEM-EDX result), the extinction coefficient at $91.6 \mathrm{eV}$ are $7.00 \times 10^{-3}$ and $7.36 \times 10^{-3}$ respectively, the latter being in agreement with the experimental values.

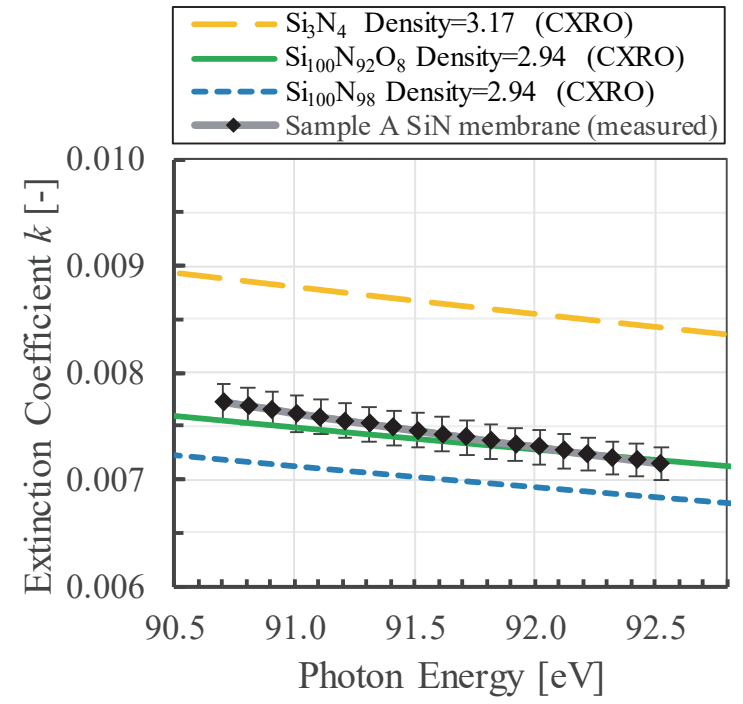

Fig. 12. Extinction coefficient $k$ for the $\mathrm{SiN}$ membrane, measured and theoretical [21].

Taking a composition of the $\mathrm{SiN}$ between the ERD and EDX results, the measured values are then slightly higher compared to the theoretical values, but the difference can be explained by the two thin oxide layers present at the interfaces of the membrane, which slightly impact (less than $3 \%$ ) the transmission measurement due to the higher absorption of Oxygen atoms. In any case, the measured value, including oxide layer, will be used as input for calculation of the photoresist extinction coefficient, and not the theoretical value, therefore avoiding the marginal error due to interfaces.

For sample B, the extinction coefficient of photoresist was calculated assuming the total transmittance is the product of $\mathrm{SiN}$ transmittance

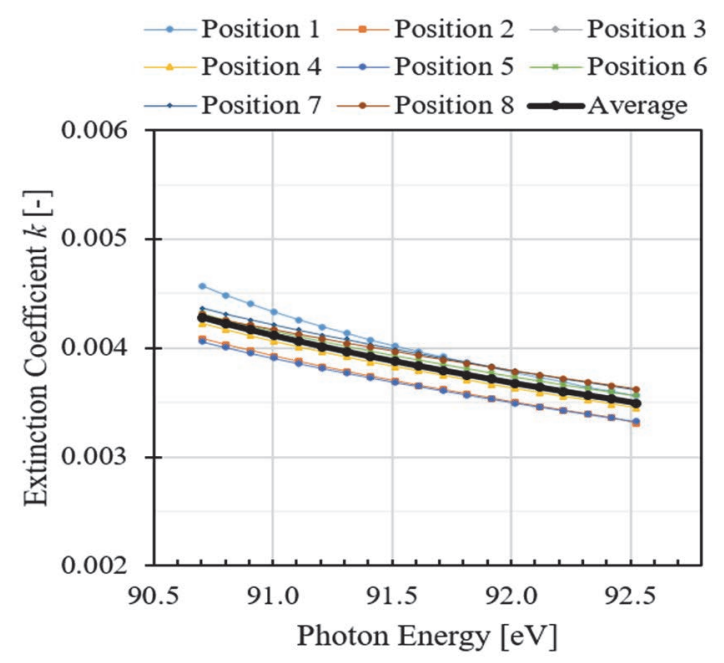

Fig. 13. Photoresist extinction coefficient as evaluated by transmittance results of Fig. 11 and using Eq. (6). 
and photoresist transmittance, thus reformulating Eq. (5):

$$
k_{P R}=\frac{\ln \left(T_{T o t}\right) \times \lambda}{4 \pi d_{P R}}-\frac{k_{S i N} \times d_{S i N}}{d_{P R}}
$$

The extinction coefficient of the photoresist $k_{P R}$ was thus obtained taking $d_{P R}$ and $d_{S i N}$ as calculated using the interpolated thickness values given in Fig. 10 and $k_{S i N}$ as calculated from sample A. The results for photoresist extinction coefficient are shown in Fig. 13.

Using the data in these figures, the extinction coefficient of the photoresist at EUV wavelength $(13.53 \mathrm{~nm} / 91.6 \mathrm{eV})$ can be calculated taking the average of the 8 measurement positions. For the photoresist tested, a value of $k_{P R}$ of $3.8 \pm 0.3 \times 10^{-3}$ was finally obtained.

\section{Conclusion}

In this article an efficient and accurate process for the evaluation of EUV photoresist extinction coefficients has been developed. Photoresist thin film layers on top of a free-standing SiN membrane have been prepared successfully. We recommend a sample size of $4 \times 4 \mathrm{~mm}^{2}$ to limit fragility of the membrane while having sufficient area allowing measurements at various positions. Further, characterization of these ultra-thin $\mathrm{SiN}$ and photoresist membranes has been performed allowing to measure thicknesses accurately, increasing the precision of the extinction coefficient calculation. Soft X-ray transmission measurements have been performed for two samples. The experimentally obtained extinction coefficient of Si-rich silicon nitride is $7.4 \pm 0.2 \times 10^{-3}$ which is in good agreement with the CXRO theoretical values, validating the accuracy of the procedure. The extinction coefficient of the photoresist tested is $3.8 \pm 0.3 \times 10^{-3}$. This technique permits an experimental measurement of the absorption coefficient of thin films, which will allow the comparison of the absorption coefficient for various photoresists platforms, without the need of their composition or density. The information gathered thanks to this method can thus be helpful for EUV photoresist research and development.

\section{Acknowledgement}

A. Rathore and Y. Vesters acknowledge the financial support from Imec through collaboration with KU Leuven. The authors would like to acknowledge technical support from Elettra synchrotron research facility, Italy. The authors also thank K.V. Luong for his help with the XRR data collection and treatment, Nadia Vandenbroeck for the support in X-SEM measurement, and the MCA team for STEM measurement.

\section{References}

1. H. J. Levinson and T. A. Brunner, Proc. SPIE, 10809 (2018) 1080903.

2. A. Mallik, J. Ryckaert, A. Mercha, D. Verkest, K. Ronse, and A. Thean, Proc. SPIE, 9422 (2015) 94221N.

3. G. D. Hutcheson, Proc. SPIE, 10583 (2018) 1058303.

4. G. M. Gallatin, P. Naulleau, D. Niakoula, R. Brainard, E. Hassanein, R. Matyi, J. Thackeray, K. Spear, and K. Dean, Proc. SPIE, 6921 (2008) 69211E.

5. O. R. Wood and H. Kinoshita, "EUV Lithography", SPIE Press, Belligham (WA), (2018) p33.

6. Y. Kwark, J. Braco-Vasquez, M. Chandhok, H. Cao, H. Deng, E. Gullikson, and C. K. Ober, J. Vac. Sci. Technol. B, 24 (2006) 1822.

7. B. L. Henke, E. M. Gullikson, and J. C. Davis, At. Data Nucl. Data Tables, 54 (1993) 181.

8. D. De Simone, S. Sayan, S. Dei, I. Pollentier, Y. Kuwahara, G. Vandenberghe, K. Nafus, M. Shiratani, H. Nakagawa, and T. Naruoka, Proc. SPIE, 9776 (2016) 977606.

9. Y. Vesters, J. Jiang, H. Yamamoto, D. De Simone, T. Kozawa, S. De Gendt, and G. Vandenberghe, J. Micro/Nanolith. MEMS MOEMS, 17 (2018) 043506.

10. Y. Fukushima, T. Watanabe, T. Harada, and H. Kinoshita, J. Photopolym. Sci. Technol., 22 (2009) 85.

11. J. Jiang, D. De Simone, and G. Vandenberghe, Proc. SPIE, 10146 (2017) 101460A.

12. D. De Simone, Y. Vesters, and G. Vandenberghe, Adv. Opt. Technol., 6 (2017) 163.

13. CXRO database, available at http://henke.lbl. gov/optical_constants/atten2.html.

14. D. Mamezaki, M. Watanabe, T. Harada, and T. Watanabe, J. Photopolym. Sci. Technol., 29 (2016) 749.

15. R. Ohnishi, T. Watanabe, Y. Fukushima, M. Osugi, and H. Kinoshita, Jpn. J. Appl. Phys., 48 (2009) 06FA08.

16. G. D. Kubiak, E. M. Kneedler, R. Q. Hwang, M. T. Schuldberg, K. W. Berger, J. E. Bjorkholm, and W. M. Mansfield, J. Vac. Sci. Technol. B, 10 (1992) 2593.

17. R. Garg, A. Antohe, and G. Denbeaux, Proc. SPIE, 6517 (2013) $65172 \mathrm{O}$. 
18. R. Fallica, J. K. Stowers, A. Grenville, A. Frommhold, A. P. G. Robinson, and Y. Ekinci, $J$. Micro/Nanolith. MEMS MOEMS, 15 (2016) 033506.

19. K. R. Williams, K. Gupta, and M. Wasilik, J. Microelectromech. S., 12 (2003) 761.
20. M. Wormington, C. Panaccione, K. M. Matney, and K. D. Bowen, Phil. Trans. R. Soc. Lond. A, 357 (1999) 2827.

21. CXRO database, available at http://henke.lbl. gov/optical_constants/getdb2.html. 